FOUNDATIONS

ADVANCES

\title{
The revival of the Bravais lattice
}

\author{
Howard D. Flack*
}

Chimie minérale, analytique et appliquée, University of Geneva, Geneva, Switzerland. *Correspondence e-mail: crystal@flack.ch

Received 1 January 2015

Accepted 6 February 2015

Keywords: Bravais lattices; phase transformations; cell reduction; crystallographic nomenclature.

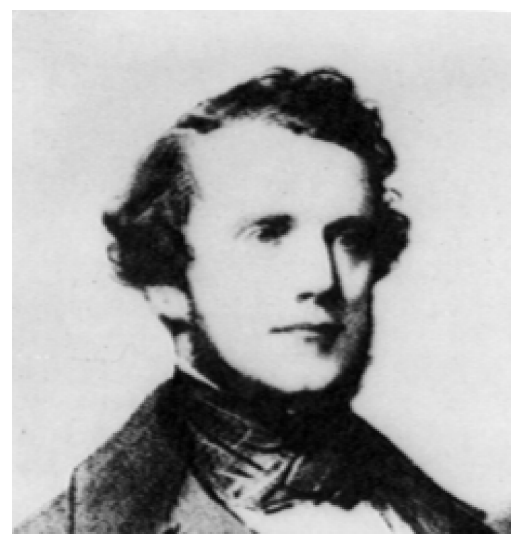

(C) 2015 International Union of Crystallography
The 14 Bravais-lattice types are at the very heart of crystallography, learned in Chapter 1 of any introductory course on the topic. It is somewhat remarkable that, in the second decade of the 21st century, we may still learn new things about them, but Hans Grimmer's paper Partial order among the 14 Bravais types of lattices: basics and applications (Grimmer, 2015) does this and provides us with important new insights. It presents an entirely original way of determining the hierarchical arrangement of Bravais-lattice types. The result is summarized in an easily understood figure (Fig. 2 in the paper, reproduced here also as Fig. 2). In the figure, the Bravais-lattice type at the upper end of a line is a special case of the type at its lower end. Grimmer's approach to determining the hierarchy is to examine the group-subgroup relations amongst the space groups of the Bravais-lattice types. The latter are those (14) symmorphic space groups with the point group of a holohedry.

Grimmer's approach yields the same result as previous analyses based on consideration of the metrical properties of lattices by way of special cases of the unit-cell dimensions. The hierarchy of the Bravais lattices is largely unknown and ignored by the crystallographic community. Implicitly, this hierarchy, and indeed the Bravais lattices themselves, are apparently considered to be of little importance and use to crystallographers. This is startling, as a fundamental property of a space group is its set of translational symmetry operations represented by the Bravais lattice. Grimmer's paper shows us how knowledge of the Bravais lattice hierarchy can help us solve real-world structures.

The following short quiz will test your knowledge and understanding of the Bravais lattice.

(1) What are the Bravais-lattice symbols of the following space groups: No. 2, No. 40, No. 150 and No. 161 ?

(2) What is the essential geometric difference between the Bravais-lattice types $m P$ and $m S$ ? Note: The correct answer contains neither the words 'affine equivalent' nor the words 'unit cell'. Try also the Bravais-lattice types $o P, o S, o F$ and $o I$.

Take a look at Fig. 8.2.1.1 of International Tables for Crystallography, Volume A, Space-Group Symmetry (2002) (hereafter ITA), which is reproduced here as Fig. 1. It shows the classifications of space groups. In the upper part of the diagram, the classification splits into two separate branches. On the right-hand branch, there is a classification in terms of the point group of the space group indicated as the ' 32 (geometric) crystal classes'. This classification is greatly loved by mineralogists and those interested in the physical properties of crystals. The banner heading of each space group in Part 7 of ITA clearly indicates its (geometric) crystal class, although it is easy to derive this from the space-group symbol itself. On the left-hand branch of Fig. 8.2.1.1 there is a classification in terms of the lattice translations indicated as '14 Bravais flocks'. This classification has far fewer enthusiasts. The banner heading of each space group in Part 7 of ITA does not indicate its Bravais-lattice type. I have often been told that it is 'easy' to derive the Bravais-lattice type from the other information available for the space group in ITA. My translation into English of this declaration is that if you know the result, it is easy to derive it. How did you get on with question (1) of the quiz?

The upper part of Fig. 8.2.1.1 contains the rather coarse classifications of the space groups into ' 6 crystal families', '7 lattice systems' and '7 crystal systems'. The 'crystalsystem' classification arose from mineralogists in the 18th and 19th centuries who had no knowledge of space groups. The '7 lattice systems' and '7 crystal systems' are different but very similar. In those situations where they differ, one finds considerable confusion in 


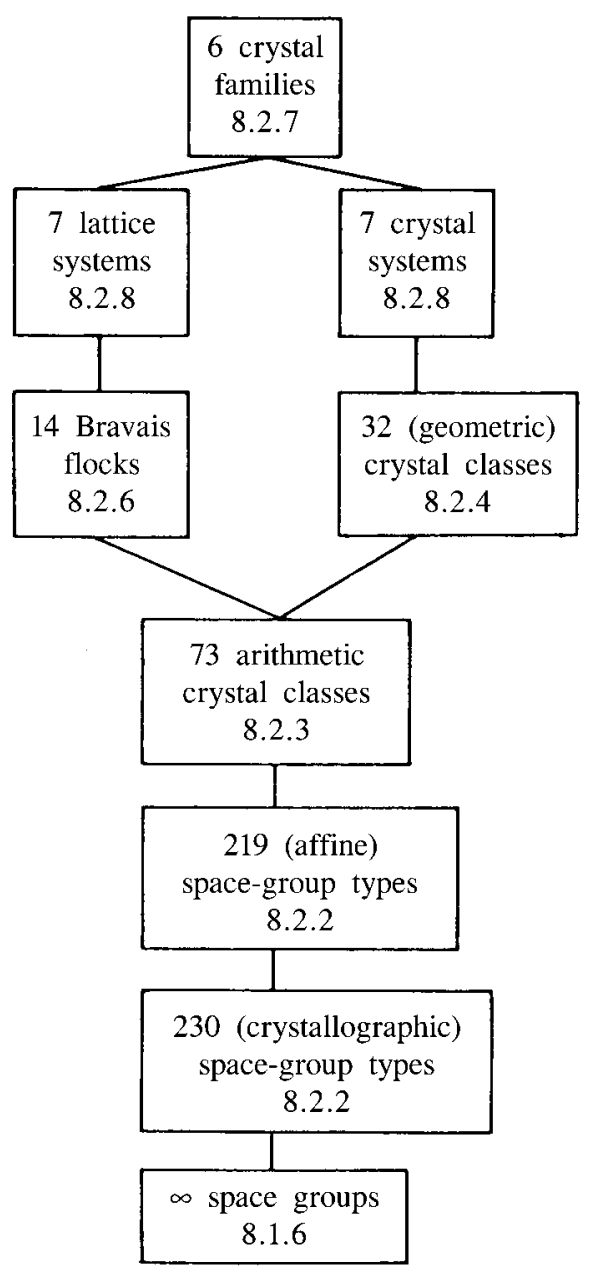

Figure 1

Classifications of space groups. In each box, the number of classes, e.g. 32, and the section [of ITA] in which the corresponding term is defined, e.g. 8.2.4, are stated. [Fig. 8.2.1.1 of International Tables for Crystallography, Volume A, Space-Group Symmetry. Copyright (2002) International Union of Crystallography. Reproduced with permission.]

their use. The 'crystal-family' classification avoids this confusion. The time has come to drop the 'lattice-system' and 'crystal-system' classifications.

Further down Fig. 8.2.1.1 the two branches rejoin in the '73 arithmetic crystal classes'. These provide a classification of the space groups jointly according to Bravais-lattice type and crystal class. A. J. C. Wilson, in his post-retirement days at the Cambridge Crystallographic Data Centre, found that it was most advantageous to classify crystal structures simultaneously on the Bravais-lattice type and the crystal class. Later he found out that the arithmetic crystal class was a well established concept! The approved symbol for an arithmetic crystal class is that of a symmorphic space group with the lattice symbol and point-group operations inverted. For example, $222 F, m m 2 P$ and $\overline{4} 2 m I$ are the symbols of three arithmetic crystal classes. However, as with all International

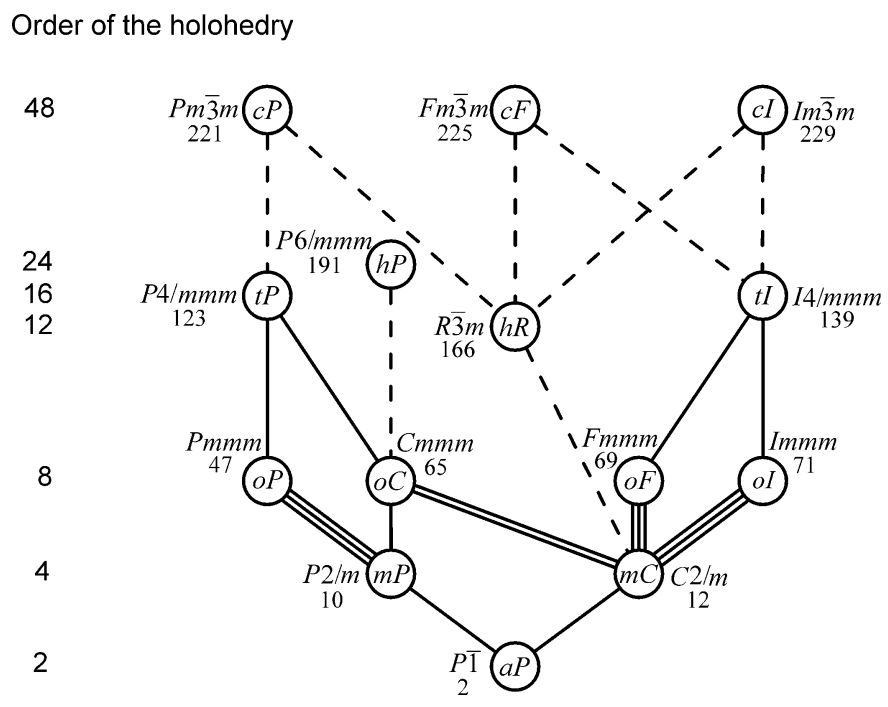

Figure 2

The Bravais type of the three-dimensional lattice at the upper end of a line is a special case of the type at its lower end. Solid lines indicate normal subgroups, dashed lines sets of conjugate subgroups. The number of conjugate groups in a set is equal to the subgroup index, i.e. the quotient of the orders of the corresponding point groups (4 for the transition cubic to rhombohedral and 3 in the other cases). [Fig. 2 of Grimmer (2015), reproduced with permission.]

symbols for space groups, the Bravais-lattice type is far from evident. Perhaps the symbols $o F 222$, oPmm 2 and $t I \overline{4} 2 m$, in which the symbol for the Bravais lattice is followed by that of the crystal class, would have achieved a greater clarity. The same applies to the space-group symbols themselves. Indeed, the section Experimental details used in the journals of the International Union of Crystallography contains an entry 'Crystal system, space group', an implicit assertion that, somehow or other, the space-group symbol by itself does not contain clearly all of the information required. Instead of 'Monoclinic, $P 2_{1} / n$ ' might not ' $m P 2_{1} / n$ ' have been a clearer and more compact symbol?

Grimmer himself gives an example of the application of the hierarchy of Bravais-lattice types in solid-state physics. Another one of practical use to the structure analyst is in the determination of the space group of a crystal. The first stage of this process consists in the determination by cell reduction of the observed Bravais-lattice type from the positions of the Bragg reflections in the diffraction data. One chosen Bravaislattice type may be very close metrically to another. Grimmer's Fig. 2 shows how one Bravais-lattice type is related to its closest metric neighbours. Fig. 2 should be on every structure analyst's desk.

\section{References}

Grimmer, H. (2015). Acta Cryst. A71, 143-149.

International Tables for Crystallography (2002). Volume A, SpaceGroup Symmetry, 5th ed., edited by Th. Hahn. Dordrecht: Kluwer. 